\title{
SKALPEL-ICT: SIMULATION KERNEL APPLIED TO THE PLANNING AND EVALUATION OF IMAGE-GUIDED CRYOTHERAPY*
}

\author{
ALEXANDRA BRANZAN ALBU \\ Computer Vision and Systems Lab, Laval University, Québec (QC),G1K 7P4, Canada \\ DENIS LAURENDEAU \\ Computer Vision and Systems Lab, Laval University, Québec (QC),G1K 7P4, Canada \\ CHRISTIAN MOISAN \\ Dept. of radiology, Laval University, Québec (QC),G1K 7P4, Canada \\ DENIS RANCOURT \\ Dept. of Mechanical Engineering, Sherbrooke University, Sherbrooke (QC), J1K 2R1, \\ Canada
}

\begin{abstract}
The SKALPEL-ICT project (Simulation Kernel Applied to the Planning and Evaluation of Image-guided Cryotherapy) aims at the developing of a virtual environment for the simulation of liver cancer cryotherapy. This simulator will mainly be used to plan the configuration and the positioning of the cryo-probes for an image-guided cryo-surgical intervention. Moreover, the prototype of the simulator will constitute an excellent training tool for future surgeons. The design of a simulator of this type involves several challenges related to the multidisciplinary nature of the project, which imposes the use of a modular approach. Presently, we are developing simultaneously three parallel models in the SKALPEL-ICT project: the thermal model associated with the prediction of the temperature of an ice-ball, the geometric model associated with the 3D reconstruction of hepatic tumours and the mechanical model which reproduces the real deformations of the liver and force feed-back during the cryo-probe insertion process. The final phase of the project is to deal with the integration of these three models into a virtual environment using an interaction-centric modeling approach.
\end{abstract}

\section{Introduction}

The virtual environment technologies offering support for medical applications are mainly designed for diagnosis, education, training, and rehabilitation. Whereas surgical training traditionally involves one-on-one experiences following a master-apprentice model, virtual environments dedicated to surgery simulation allow the trainees to learn new procedures, practice skills several

\footnotetext{
* This work is supported by the strategic grant no. 234773 of the National Sciences and Engineering Research Council of Canada.
} 
times, and eventually assess their level of competence before they operate on real patients. Moreover, the rather recent development of minimally invasive interventional techniques (endoscopy[1], eye surgery, cryotherapy etc.) triggered an increased interest in the area of computer-based medical simulators.

This paper describes the development of a virtual environment for the simulation of liver cancer cryotherapy. This simulator will be used to plan the configuration and the cryo-probes for an image-guided cryo-surgical intervention, and to visualize the thermal information corresponding to the intra-operative freezing process as well. Moreover, the prototype of the simulator will constitute an excellent training tool for future surgeons. The design of a simulator of this type involves several challenges related to the multidisciplinary nature of the project, which imposes the use of a modular approach. The following three sections of this paper describe the geometric model associated with the segmentation and $3 \mathrm{D}$ reconstruction of hepatic tumours, the mechanical model which reproduces the real deformations of the liver and force feed-back during the cryo-probe insertion process, and the thermal model associated with the prediction of the temperature inside the growing ice-ball. Section 5 gives details about the final integration of these three models into a dynamic virtual environment. The last section of this paper outlines the conclusions of our research.

\section{Geometric modeling}

\subsection{Segmentation}

The database for the geometric modeling consists of axial, saggital and coronal sequences of 2D magnetic resonance images corresponding to parallel and equidistant anatomical slices. In every acquisition plane, a large difference is noted between the horizontal intra-slice resolution (ca. $1.56 \mathrm{~mm}$ ) and the vertical inter-slice resolution (ca. $10 \mathrm{~mm}$ ). This difference cannot be minimized during the acquisition process, due to the respiratory motion artefact and to technical limitations. Therefore, 3D segmentation approaches are not reliable in our case and we must consider 2D segmentation techniques. We concentrate on two particular features of abdominal MRI data. The first one is the inhomogeneous texture of the liver tumours at a certain stage of their evolution (see Figure 1). For instance, large-sized liver tumours develop a lobular appearance due to the aggregation of several small-sized lesions surrounded by edema. The second feature is the non-uniform sharpness of the tumour boundary which may contain sharp segments alternating with "blurred" segments (see Figure 1). Due to infiltration into surrounding tissues, malignant liver lesions often present contours that do not reveal a clear-cut transition.

To detect the region of interest, we create an isolabel tumour contour map using a multi-threshold technique and a similarity measure for the contours 
(see Figure 1b). In order to extract the isolabel contour map of the tumour, we need a minimum amount of information about its location in the liver. The radiologist is asked to select one single reference pixel located inside the tumour. Tumours with "blurred" contours of variable sharpness are detected with a pixel aggregation algorithm based on local texture information [2] (see Figure 1c).
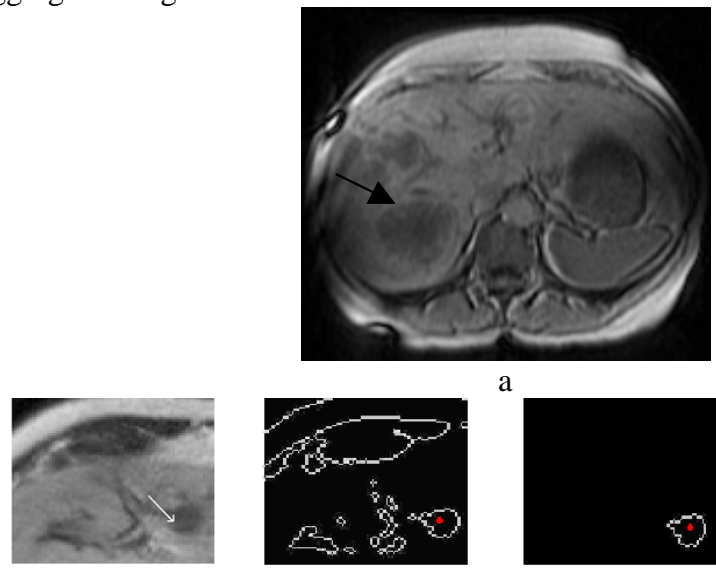

a
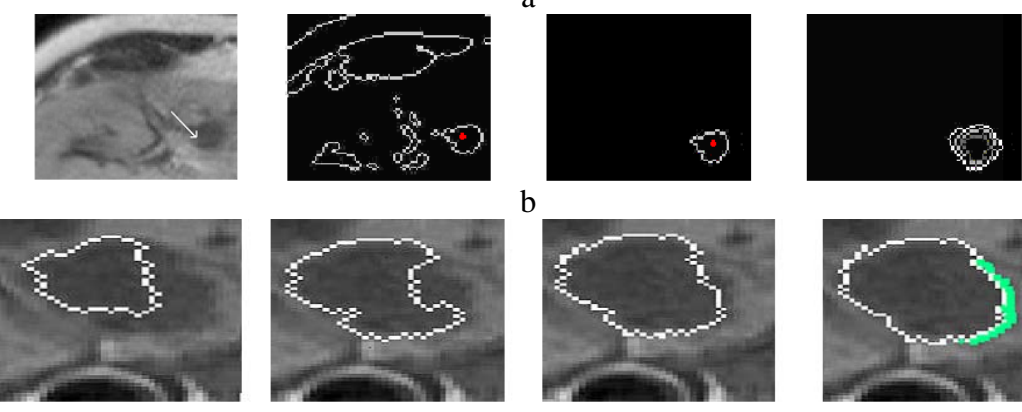

b
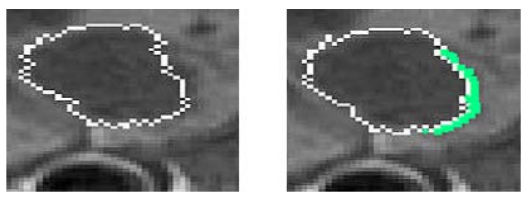

Figure 1. a) Axial image containing several hepatic lesions. The arrow points at a tumour with nonuniform texture and "blurred" boundaries; b) Extraction of the isolabel contour map corresponding to a tumour containing the red starting pixel; c) Evolution of the pixel aggregation algorithm. The green area represents the tumour region which has not been detected.

\subsection{Reconstruction}

Segmentation results are input data for a custom 3D reconstruction algorithm [3], which combines shape-based interpolation and contour-based extrapolation (see Fig. 2a and 2b). While interpolation generates intermediate slices between pairs of adjacent input slices, extrapolation performs a smooth closing of the external surface of the model. Surface rendering consists in the generation of a triangular mesh using a parametric representation of 2-D intermediate contours. 


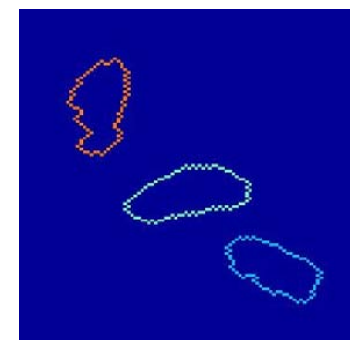

a

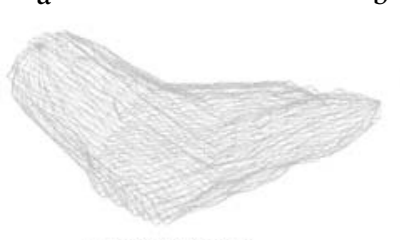

undeformed mesh

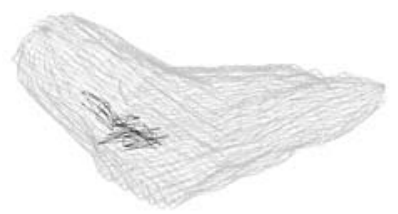

$\mathrm{dz}=4 \mathrm{~mm}$

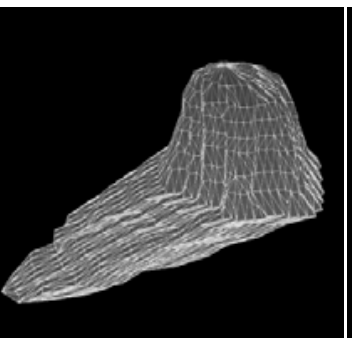

b

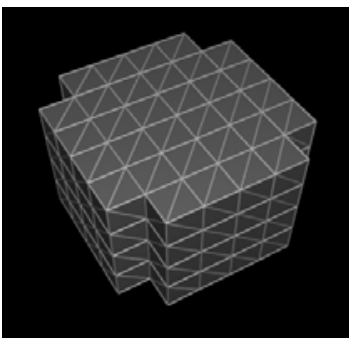

c

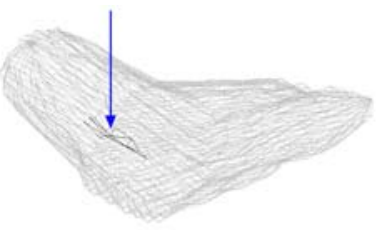

$\mathrm{dz}=2 \mathrm{~mm}$

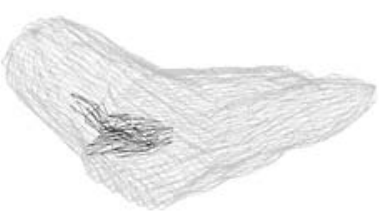

$\mathrm{dz}=6 \mathrm{~mm}$

d

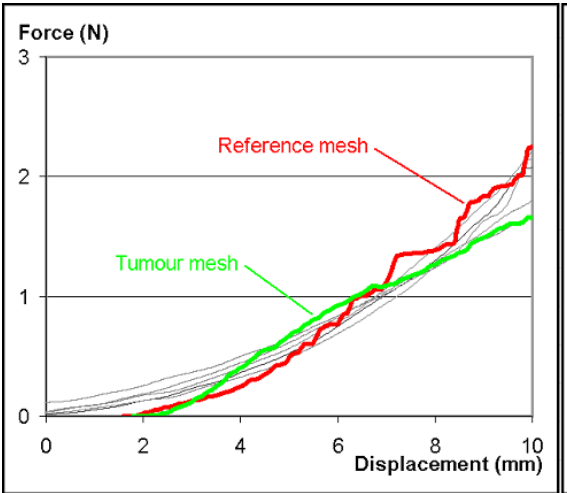

e

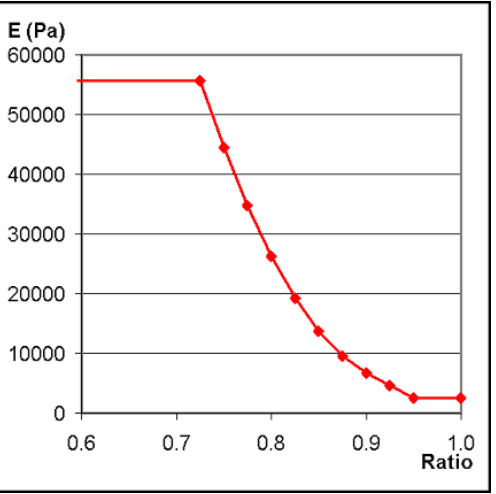

$\mathrm{f}$

Figure 2. a) Input configuration for the $3 \mathrm{D}$ reconstruction, viewed in the $\mathrm{z}$ direction; b) geometric model of the tumour corresponding to the input configuration in a); c) Reference mesh; d) Simulating the deformations during the cryo-probe insertion on the reconstructed tumour mesh; e)Five independent experimental measurements of forces during the compression of an ex vivo deer liver sample by a biopsy needle (light grey curves), and simulated forces on the reference mesh and on a reconstructed 3D tumour model. f) Mechanical law derived from experimental characterization. 


\section{Mechanical modeling}

Our mechanical model is based on the finite element based tensor-mass algorithm [4], which computes forces from a combination of local stiffness tensors attached to every mesh element. These tensors depend only on the mesh geometry at rest, and on the mechanical properties of the tissue. Therefore they can be computed in a preliminary step, while computation in the actual simulation is limited to a linear combination of stiffness matrices and displacement vectors, meeting real-time constraints.

As shown in [5], it is possible to extend the linear tensor-mass model for simulating different types of non-linear and visco-elastic mechanical properties. The first results were obtained using meshes consisting of a regular assembly of cubic elements (see Figure 2c). The same mechanical model can be applied on a non-uniform mesh derived from a reconstructed 3D tumour model (see Figure 2b). The mechanical tissue properties used for testing were obtained from experimental in vitro compression on deer liver membrane by a biopsy needle, since no in vivo mechanical data of liver tumour could be measured so far.

Although differences can be observed between the two simulations due to the different mesh geometries, accordance between experimental data and both simulations can be considered satisfactory. Due to the thin tumour geometry (approximately $8 \mathrm{~mm}$ in thickness), forces on the tumour mesh tend to increase more slowly at higher deformations, as it becomes almost entirely pierced. This comparison shows that the proposed mechanical model can be successfully applied to variable geometries. Figure $2 \mathrm{e}$ presents five independent experimental force measurements, as well as the simulated force on the reference mesh used to fit our model parameters, and the simulated force computed on the non-uniform tumour mesh. Compression speed was in all cases constant at $10 \mathrm{~mm} / \mathrm{s}$.

The tissue model derived from these measurements was highly non-linear. Figure 2f shows the non-linear function introduced into the tensor-mass model to account for these properties. For low deformations the Young modulus was $E=$ $3600 \mathrm{~Pa}$, and the Poisson coefficient was kept constant at $v=0.4$.

Figure $2 \mathrm{~d}$ shows several deformed mesh configurations at different time steps.

\section{Thermal modelling}

When performing the cryo-surgical intervention, the surgeon must precisely determine the extent of cell destruction induced by the cryogenic probes. Obviously, the goal is to entirely destroy the malignant tissue while avoiding to harm adjacent healthy tissue. While, temperatures between $-40^{\circ} \mathrm{C}$ and $-60^{\circ} \mathrm{C}$ are considered to be effective lethal temperatures for a wide range of in vitro and in vivo tissues [6], magnetic resonance does not exist below $0^{\circ} \mathrm{C}$. 
Due to the lack of accurate thermal information during MR image-guided cryotherapy procedures, the hold time and the number of freeze/thaw cycles are in most of the cases estimated empirically. The goal of the thermal module is to enhance real-time MR images with quantitative data on the growth rate of the iceball volume and tissue temperature during the procedure. This additional information should provide a better control of the cryo-surgical intervention, since it enables the quantification of the effect of a chosen freeze-thaw cycle.

The most straightforward mapping of the thermal evolution during cryo-surgery implants a set of thermosensors at specific landmarks. While this approach provides a direct reading of local temperatures, the spatial resolution of its measurements is significantly coarse. In contrast, MRI combined with the energy equation of the freezing process can be used to compute the thermal map.

We have developed a simple analytical method [7], which uses experimental measures and numerical processes to compute thermal maps with good spatial and thermal resolutions. The temperature estimation procedure contains the following four main steps.

1) Image processing. Images acquired during the freezing cycle are subtracted from a reference image and normalized afterwards. This scaling is used for emphasizing intensity variations inside the growing iceball region.

2) Intensity-to-Temperature Correlation. The relationship was directly measured between the normalized intensity of the MR signal and the temperature of a given location within the iceball by means of thermosensor readings.

3) Intensity and thermal segmentations. The volumes and diameters of the iceball are extracted from the normalized images and are afterwards represented as functions of time and intensity. The straightforward conversion from intensity to temperature allows to substitute one for the other in the distributions.

4) Regressions in Time and Thermal Domains. The volume and diameter distributions are extrapolated to lower temperatures using two simple regressions in time and temperature domains respectively. Thus, it is possible to estimate temperatures at any time and at any distance away from the probe.

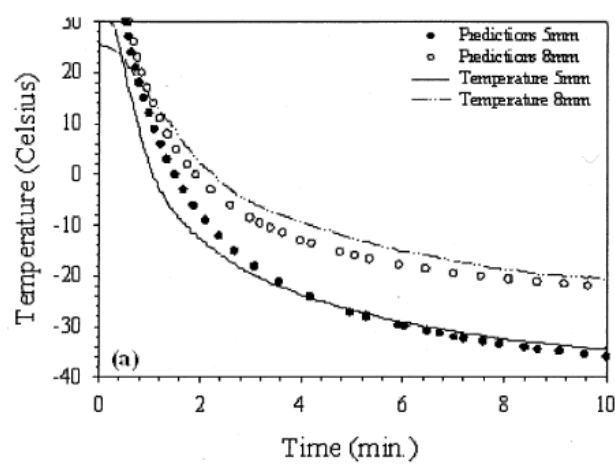

Figure 3. Measured and predicted temperatures at different distances from the cryoprobe axis [7] 
Figure 3 shows the measured and predicted temperatures at two different distances (5mm and $8 \mathrm{~mm}$ ) from the cryoprobe axis in ex vivo pig liver.

\section{Modular integration based on the APIA approach}

The final integration phase of the project uses a task-oriented simulation framework, namely the Actor-Property-Interaction Architecture (APIA) [8]. APIA implements a new paradigm named Interaction Centric Modelling that allows a flexible design of virtual entities and their physical behaviours, such as viso-elasticity, dry friction during needle insertion, etc.

Figure 4 illustrates an example using APIA in a virtual cryo-surgery context. In such a virtual world, a surgeon can make an incision with a scalpel or freeze the liver with a cryoprobe. The Cut interaction operates between the Cutable character and the Force Generator character, while the Freeze interaction occurs between a Heat Sensitive character and a Heat Generator character. These characters group some properties required by the interaction. An actor will own all the properties of inherited characters. Since the dependency between instantiated entities is generic, it is possible for the liver to inherit from a Heat Generator character and therefore take into account the fact that the liver is itself a heat generator.

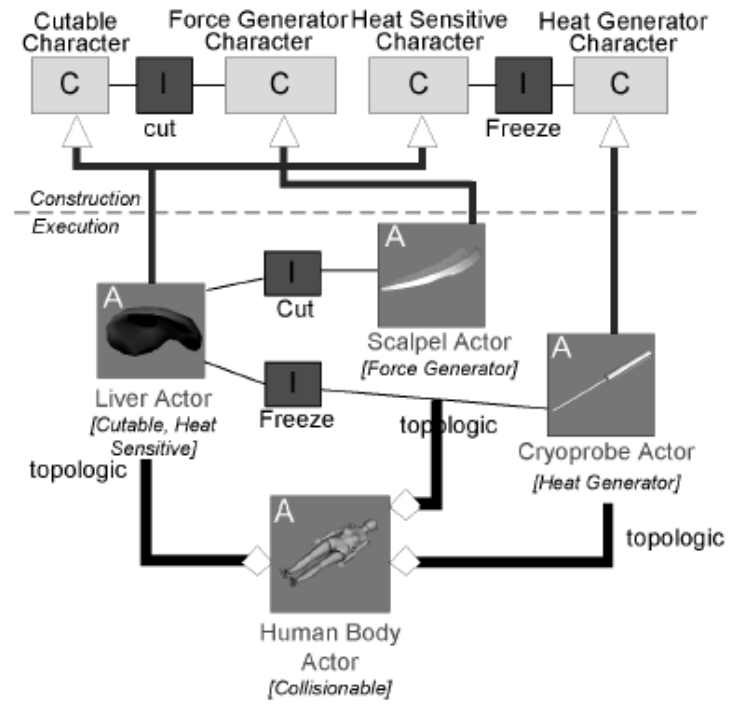

Figure 4. Representation of some virtual entities and of their interaction during a virtual cryosurgery [8] 


\section{Conclusions}

It is widely recognized that the simulation of surgery through realistic Augmented Reality (AR) environments offers a safe, flexible, and cost-effective solution to the problem of planning the treatment procedures as well as in training surgeons in mastering highly complex manipulations. In this context, SKALPEL-ICT represents a unique immersive and interactive augmented reality simulation environment for the MRI-guided cryotherapy of liver.

SKALPEL-ICT addresses the simulation of two cryosurgery steps: i) the viscoelastic behavior of the tumor during the insertion of a virtual cryoprobe and ii) the freezing process when the cryoprobe is activated. These simulations imply that a 3D geometric model of the tumor is available. This model is reconstructed from segmented tumour slices in MR images.

Unlike most simulation environments based on scene graphs, SKALPEL-ICT implements a physically-oriented simulator based on the concepts of actors, properties, and interactions. Actors are described by properties and interact with each other through interaction objects.

\section{References}

1. L. Hong, A. Kaufman, Y. Wei, A. Viswambharan, M. Wax, and Z. Liang "3D Virtual Colonoscopy ," IEEE Symposium on Frontier in Biomedical Visualization, IEEE CS Press, Los Alamitos, 1995, pp. 26-32.

2. A. Branzan Albu, D. Laurendeau, and C. Moisan, "Tumour detection in MR Liver Images by Integrating Edge and Region Information", ESSAIM Proceedings of Modelling and Simulation for Computer-Aided Medicine and Surgery, Rocquencourt, France, 2002.

3. A. Branzan Albu, J.-M. Schwarz, D. Laurendeau, and C. Moisan, "Integrating geometric and biomechanical models of a liver tumour for cryosurgery simulation", in Proc. IS4TM : International Symposium on Surgery Simulation and Soft Tissue Modelling, (Juan-Les-Pins, France), juin 12-13 2003.

4. S. Cotin, H. Delingette, and N. Ayache, "A hybrid elastic model for real-time cutting, deformations, and force feedback for surgery training and simulation", Visual Computer, Vol. 16, 2000, pp. 437-452.

5. J.-M. Schwartz, E. Langelier, D. Rancourt, C. Moisan, and D. Laurendeau, "Nonlinear soft tissue deformations for the simulation of percutaneous surgeries", Proc. MICCAI 2001, Utrecht, Netherlands.

6. J. Baust, A. A. Gage, H. Ma, and C. M. Zhang, "Minimally Invasive CryosurgeryTechnological Advances", Cryobiology, vol.34, 1997, pp. 373-384.

7. R. Fournial, A. S. Traoré, D. Laurendeau, and C. Moisan, "An analytic method to predict the thermal map of cryosurgery iceballs in MR images", IEEE Trans. On Med. Imaging, vol.23, no.1, 2004, pp. 122-129.

8. F. Bernier, D. Poussart, D. Laurendeau, and M. Simoneau, "Interaction-centric Modelling for Interactive Virtual Worlds: The APIA approach", Proc. ICPR 2002, (Quebec, Canada), Aug. 11-15 2002. 